\title{
Finite Element Analysis of the Achilles Tendon While Running
}

\author{
Anițaș R, Lucaciu DO \\ Department of Orthopedics, "luliu Hațieganu" University of Medicine and Pharmacy, Cluj Napoca, Romania
}

\begin{abstract}
Introduction: The Achilles tendon is the most frequent recipient of traumatic injuries. The aim of this study is to identify and describe the varying load at ankle level and especially at the Achilles tendon's insertion on the calcaneus.

Methods: We conducted a finite element analysis of the Achilles tendon while running, with the aim of revealing maximal loads and strains during a step in a running sequence. A 3D model of the Achilles tendon was built, based on MRI slides of a healthy, injury-free subject, who was asked to run over a force plate in 50 iterations. We used the recorded data to establish maximum loads and strains.

Results: We noticed a quick rise of the intra-tendinous load, from almost negligible while airborne and on first ground contact, to roughly 40 MPa in the pre-airborne phase, with possible implications in both treatment and post-injury recovery of Achilles tendon lesions.

Conclusions: Our results suggest that while early weight bearing and early exercise routines are a modern approach, care must be given in increasing the loads on the recovering region.
\end{abstract}

Keywords: FE analysis, Achilles tendon, 3D model, maximum load, strain

Received: 28 November 2012

\section{Introduction}

The Achilles tendon bears high loads repeatedly during a day's worth of movement, being the most frequent recipient of traumatic injuries due to acute or habitual overloading, a direct consequence being the acute traumatic total tendon rupture. Intra-tendinous loads can reach as high as 1400 $2600 \mathrm{~N}$ while walking, 3100-5500 $\mathrm{N}$ while running [1,2]. The current study aims to identify and describe the varying load at ankle level and especially at the Achilles tendon's insertion on the calcaneus. Breaking down the motion of this tendinous insertion site into its component forces during everyday movements is an important step in the analysis of the causes that favour, facilitate or even directly induce tears of the Achilles tendon and related structures.

The tendon is particularly designed to transfer the tensile forces from muscle to bone, although its structure does not permit it to endure excessive shearing and compression forces [3]. At rest, the tendon fibres appear in a wavy configuration [4], which disappears when the stretching passes $2 \%$ length [5]. The tendon's behaviour is considered elastic for tensile forces that stretch it no longer than $2 \%$ and hyper-elastic past this elongation. Structural damage is observed past $4 \%$ elongation - when partial tears occur - and complete tears usually happen around $8 \%$ elongation [6,7].

In the chain of motion, the Achilles tendon acts as an interface between the muscle-tendon complex (triceps surae and its tendon) and bone (calcaneus). Thus, the forces acting upon the calcaneus during its contact with the ground (ground reaction force - GRF), with the calcaneus as the

Correspondence to: Răzvan Anițaș

E-mail: rvaan_ainitasi@yahoo.com immobile part, can be considered to impact directly upon the tendon. By recording the GRF we can obtain direct data on the motions and kinematic chains of the Achilles tendon, and using the kinematic study of the tendon we were able to identify the moments in the chain of motion that involve the highest chance of injury to the tendon or its insertion site on the calcaneus.

Forces acting at foot level can be measured through a force plate, a device that takes advantage of Newton's Third Law of Motion (which states that for every applied force there is a force of similar value applied in the opposite direction). By recording the ground reaction force during various movements (such as running, walking, jumping and sidestepping) we can determine the forces that impact on the Achilles tendon during everyday motion, and in particular those originating at the tendon-bone insertion interface.

For the purpose of the study, we built a 3D model of the calcaneus and Achilles tendon, using 2D MRI slides. While arduous, the process is easily repeatable for any number of subjects and if done properly, can achieve high accuracy.

\section{Material and methods}

The necessary geometry was built starting from sagittal MRI slides, with $3 \mathrm{~mm}$ sequencing. The subject was a 23 year old male, $182 \mathrm{~cm}, 76 \mathrm{~kg}$, with no history of tendon injury or chronic ingestion of toxics (alcohol, corticosteroids, etc.), and no specific physical training. Segmentation and an initial 3D model were obtained with the help of 3DSlicer (@www.slicer.org).

Shell models thus obtained were further processed with SolidWorks 10.0, rendering into solid bodies. Finite ele- 


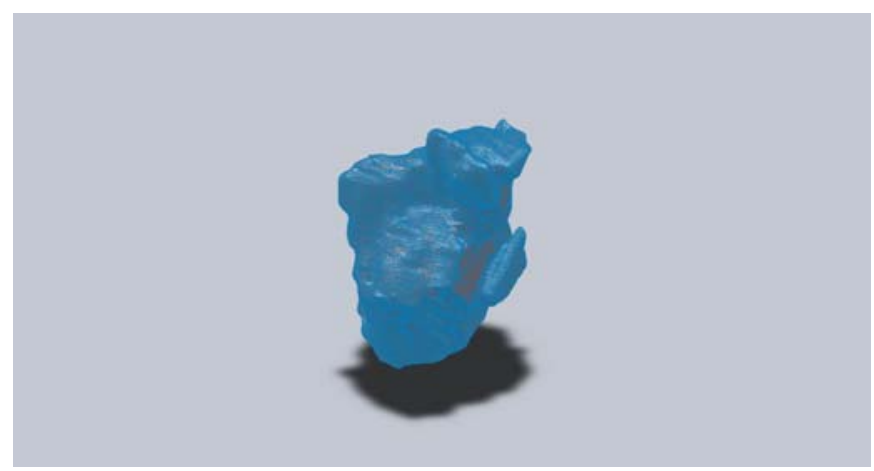

Fig. 1. FE Calcaneus model

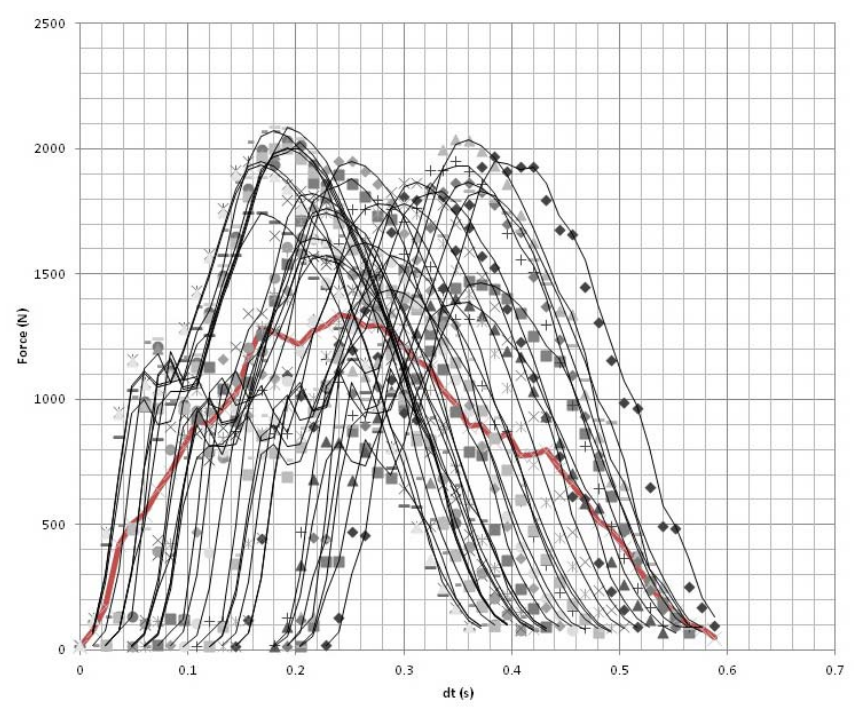

Fig. 3. Running sequence data

ment analysis was conducted with the Altair Hyperworks 10.1 Abaqus solver.

In order to emulate the calcaneus-tendon interface, the rigid body-flexible body option was used while solving. Compressive rigid was used at the interface in an attempt to simulate cartilaginous structure. Bony structures were considered homogenous, isotropic and linearly elastic materials, Young modulus and Poisson's ratio were considered to be $7300 \mathrm{MPa}$ and 0.3 respectively [8]. Compressive surfaces were given $1 \mathrm{MPa}$ Young modulus and a 0.4 Poisson's ratio [9].

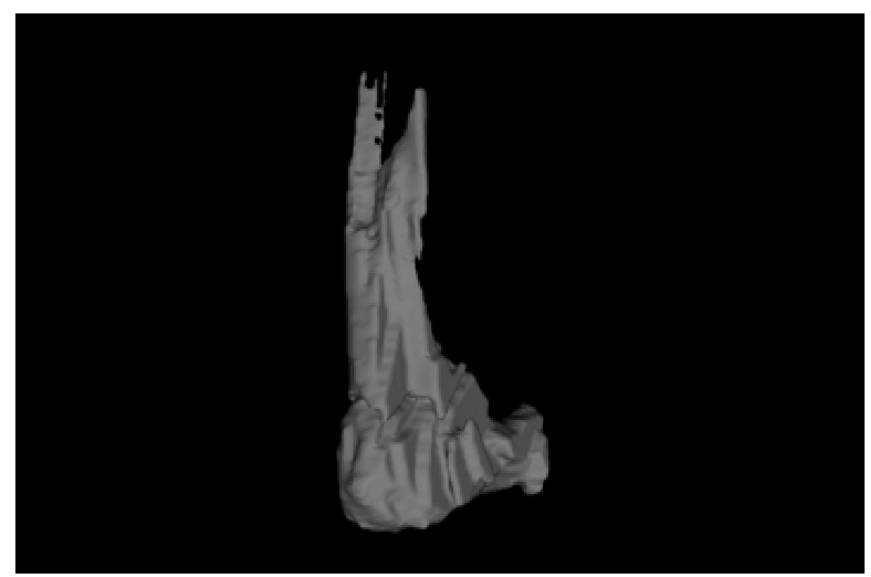

Fig. 4. 3D model of Achilles tendon and calcaneus

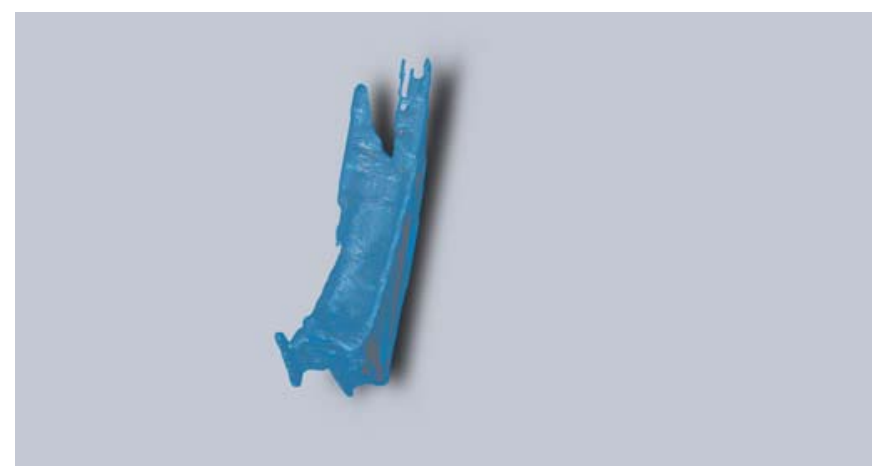

Fig. 2. FE Achilles tendon model

Achilles tendon material was emulated by considering it a hyper-elastic, incompressible material [10], defined by $\mathrm{E}=\mathrm{c}_{10}\left(\mathrm{I}_{1}-3\right)+\mathrm{c}_{01}\left(\mathrm{I}_{2}-3\right)+1 / \mathrm{D}(\mathrm{Je}-1) 2$, where $\mathrm{E}$ is kinetic energy, $\mathrm{c}_{10}, \mathrm{c}_{01}$ material constants that express deformation and $\mathrm{D}$ is a given incompressibility variable. $\mathrm{I}_{1}=\lambda_{1}{ }^{2}+\lambda_{2}{ }^{2}$ $+\lambda_{3}{ }^{2}$ and $\mathrm{I}_{2}=\lambda_{1}{ }^{-2}+\lambda_{2}{ }^{-2}+\lambda_{3}{ }^{-2}$ are first and second deviatory strain invariants defined, and a Mooney-Rivlin [11] formulation is thus defined.

Collected running sequence forces were used from a 30 -iteration running exercise. Data was obtained using a Kistler Force Plate (with a $2.5 \mathrm{kHz}$ recording frequency).

\section{Results}

A final 3D model was built, with an increased resolution, aimed at the Achilles tendon's insertion on the calcaneus, as shown in Figure 4. The von Misses stress distribution in the ankle region reveals the areas of maximal load and

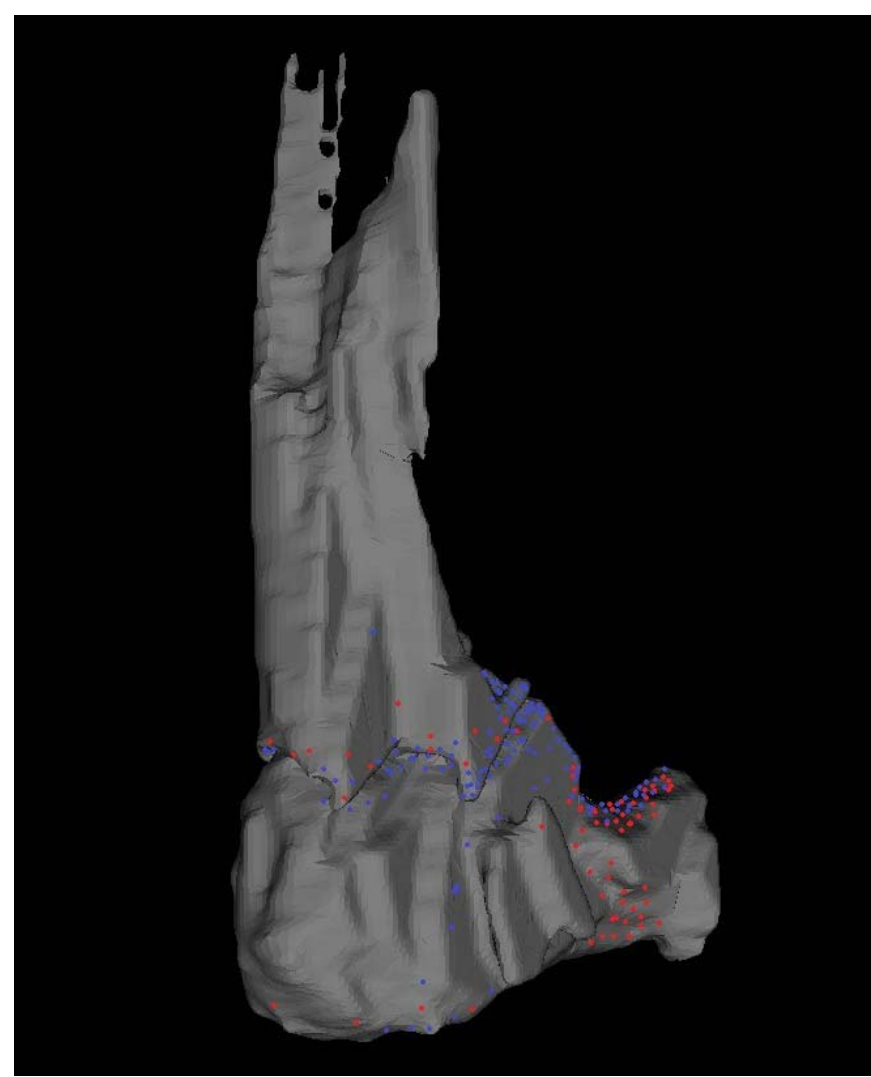

Fig. 5. von Misses stress during ground contact 


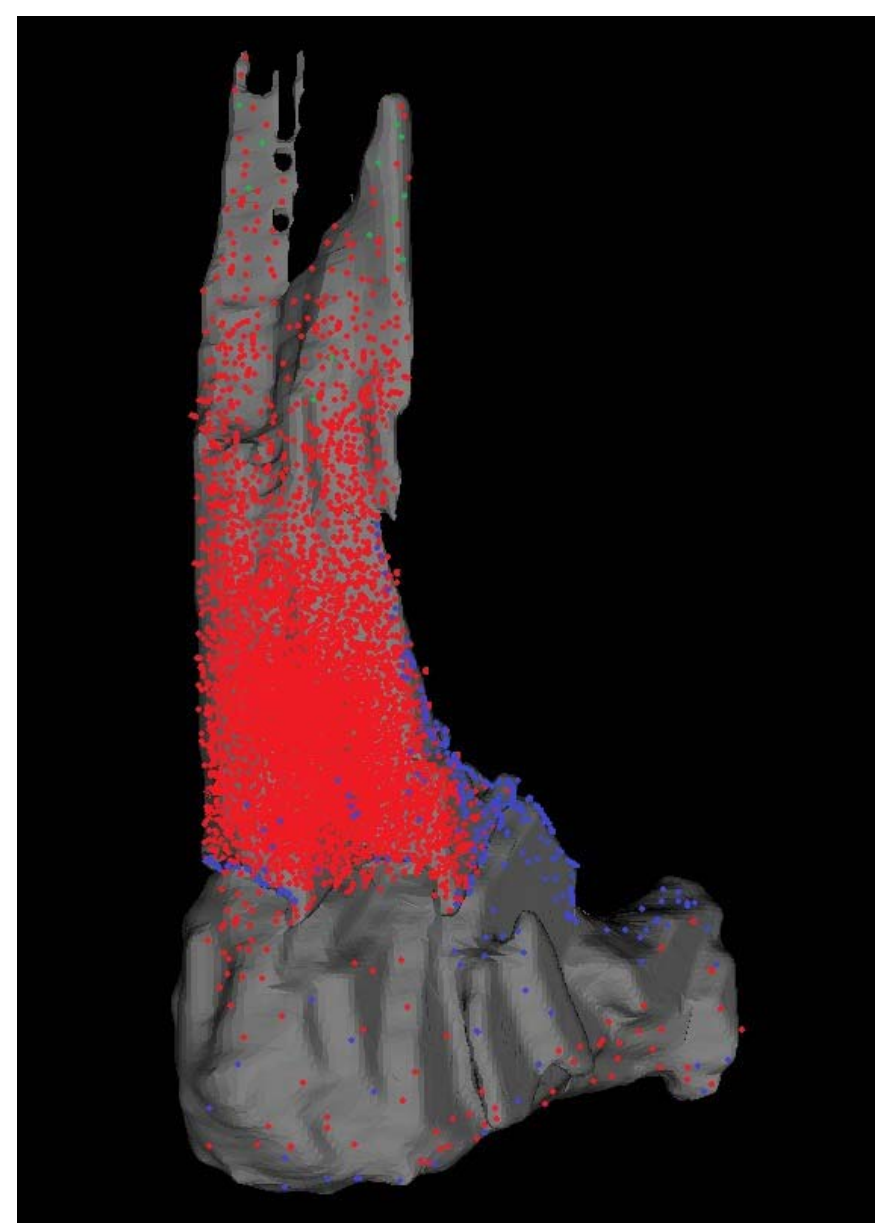

Fig. 6. von Misses stress at full traction, pre-airborne phase

the strain distribution pattern, as shown in Figure 5. To note is the low level of load during initial heel contact, when a peak stress of $0.05 \mathrm{MPa}$ is recorded at the tendon insertion on the bone. On full contact and in the pre-jump phase, peak stresses reach 22.66 MPa. Maximal peak stress was $48.37 \mathrm{MPa}$ at the last contact point, when the body goes completely airborne in the running sequence Figure 6). The running step model shows the peak, parabolic-like function (Figure 7).

\section{Discussion}

The current study aimed to build a 3D model of the Achilles tendon insertion on the calcaneus and to analyse the impact of forces upon the geometry of the ankle region during a running sequence, using finite element analysis method [12,13]. Recording ground reaction forces and a 3D mesh limited to the Achilles tendon and the calcaneus were enough to properly simulate the lower foot while running, any additional elements would have provided no further detail to the analysis [14].

Traditionally, the most frequent Achilles tendon ruptures involve the region between 3 and $6 \mathrm{~cm}$ above the calcaneal insertion of the tendon, a fact that was explained by the poor vascular support of the area. The current study reveals that the maximal loads in this region can be indeed one of the causes for acute traumatic tendon tears, even in

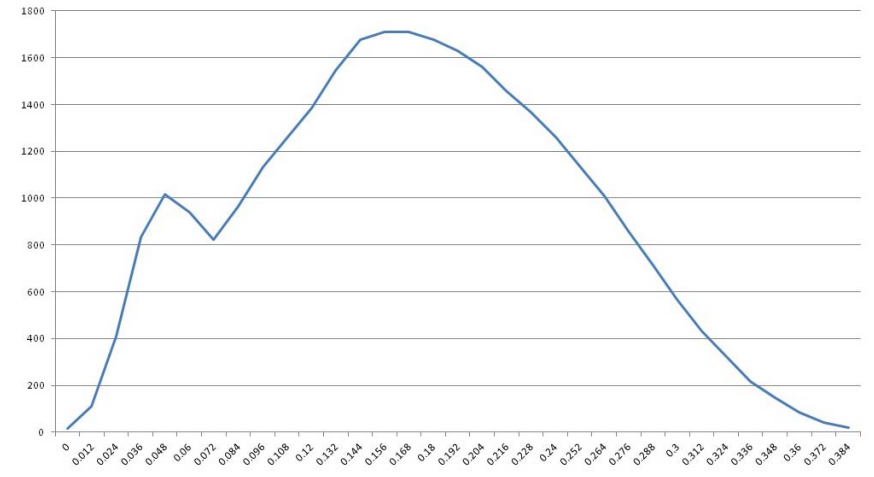

Fig. 7. Running step model

the absence of a damaged or degenerated tendon structure, especially when eccentric loading and explosive plyometric contractions are present [15].

It is worth noting the quick intra-tendinous load rises from being almost negligible while airborne and first ground contact, to roughly $40 \mathrm{Mpa}$ in the pre-airborne phase, in a very short time span [16].

Hyperaemia, shearing forces, direct hits (incidentally, the same region claims the most direct hit trauma during football matches) and torsion forces are elements that paint a striking picture of the abuse the tendon - calcaneus must bear.

\section{Conclusions}

From a therapeutic stand-point, describing an area susceptible to trauma as this is valuable in prevention as well as in recovery, especially for athletes. While early weight bearing and early exercise routines are a modern approach, care must be given in increasing the loads on the recovering region, as pointed out in the experiment. Individual biometric data becomes even more important, as forces and loads are shown to be dependent on body weight, muscle and bone length, and motion particularities.

\section{References}

1. Magnusson SP, Narici MV, Magnaris $C N$ et al. Human tendon behaviour and adaptation, in vivo. J Physiol. 2008;586(1):71-81.

2. Schileo E, Dall'Ara E, Taddei $F$ et al. An accurate estimation of bone density improves the accuracy of subject-specific finite element models. J Biomech. 2008;41(11):2483-2491.

3. Doral MN, Alam M, Bozkurt M et al. Functional anatomy of the Achilles tendon. Knee Surg Sports Traumatol Arthrosc. 2010;18:638-643.

4. Rees JD, Lichtwark GA, Wolman RL et al. The mechanism for efficacy of eccentric loading in Achilles tendon injury; an in vivo study in humans. Rheumatology. 2008;47(10):1493-1497.

5. Waggett DA, Ralphs JR, Kwan APL et al. Characterization of collagens and proteoglycans at the insertion of the human achilles tendon. Matrix Biology. 1998;16(8):457-470.

6. Lee SJ, Slleo MJ, Kremenic IJ et al. Cyclic loading of 3 Achilles Tendon Repairs Simulating Early Postoperative Forces. Am J Sports Med. 2009;37(4):786-790.

7. Azevedo LB, Lambert MI, Vaughn CL et al. Biomechanical variables associated with Achilles tendinopathy in runners. Br J Sports Med. 2009;43:288-292

8. Zhang D, Arola DD, Rouland JA. Evaluating the elastic modulus of bone using electronic speckle pattern interferometry. Exp. Tech. 2001;25:32-34.

9. Novitskaya E, Chen PY, Hamed E et al. Recent advances on the measurement and calculation of the elastic moduli of cortical and trabecular bone: a review. Theoret Appl Mech. 2011;38(3):209-297. 
10. Huang HL, Tsai MT, Lin DJ et al. A new method to evaluate the elastic modulus of cortical bone by using a combined computed tomography and finite element approach. Comp Biol Med. 2010;40(4):464-468.

11. Arnold ME, Ward SR, Lieber RL et al. A model of the lower limb for analysis of human movement. Ann Biomed Eng. 2010;38(2):269-279.

12. Tao K, Wang D, Wang $C$ et al. An In Vivo Experimental Validation of a Computational Model of Human Foot. J Bionic Eng. 2009;6(4):387-397.

13. Sun PC, Shih SL, Chen YL et al. Biomechanical analysis of foot with different foot arch heights: a finite element analysis. Computer Methods Biomech Biomed Eng. 2012;15(6), DOI:10.1080/10255842.2010.550165
14. Munteanu ES, Barton JC. Lower limb biomechanics during running in individuals with Achilles tendinopathy: a systematic review. J Foot Ankle Research. 2011;4:15.

15. Hess GW. Achilles Tendon Rupture, A Review of Etiology, Population, Anatomy, Risk Factors and Injury Prevention. Foot Ankle Spec. 2010;3(1):29-32.

16. Farris JD, Trewartha G, McGuigan MP. Could intra-tendinous hyperthermia during running explain chronic injury of the human Achilles tendon? J Biomech. 2011;44(5):822-826. 